\title{
Near-infrared reflectance bull's eye maculopathy as an early indication of hydroxychloroquine toxicity
}

This article was published in the following Dove Press journal:

Clinical Ophthalmology

19 March 2015

Number of times this article has been viewed

\author{
Keye L Wong' \\ Scott E Pautler ${ }^{2}$ \\ David J Browning ${ }^{3}$ \\ 'Retina Associates of Sarasota, \\ Sarasota, FL, USA; ${ }^{2}$ Retina Vitreous \\ Associates of Florida, Tampa, FL, USA; \\ ${ }^{3}$ Charlotte Eye Ear Nose and Throat \\ Associates, Charlotte, NC, USA
}

Correspondence: Keye L Wong Retina Associates of Sarasota, 3920 Bee Ridge Road, Building D, Sarasota, FL 34233, USA

$\mathrm{Tel}+$ I 94I 9240303

Fax + I 94I 9240309

Email keyewong@gmail.com
Importance: In some patients, hydroxychloroquine ocular toxicity may progress even following cessation of therapy. Any leverage the clinician may use to allow earlier detection may avert significant vision loss.

Observation: We report three cases suggesting that bull's eye maculopathy seen on nearinfrared reflectance with a confocal scanning laser ophthalmoscope could be an early, objective manifestation of hydroxychloroquine ocular toxicity, and with progression of the disease this near-infrared "bull's eye" change may disappear.

Conclusion and relevance: Alerting clinicians to this observation may allow a larger case series to corroborate the hypothesis that bull's eye maculopathy detected by near-infrared reflectance may represent an early sign of hydroxychloroquine toxicity.

Keywords: confocal, scanning laser ophthalmoscope, multifocal ERG

\section{Introduction}

Bull's eye maculopathy describes the ophthalmoscopic appearance of a ring of paracentral atrophy of the retinal pigment epithelium (RPE) surrounding the fovea. When associated with hydroxychloroquine (HC) toxicity, this visible manifestation as documented by fundus photography and fluorescein angiography often appears as a relatively late finding already associated with visual loss. The goal of $\mathrm{HC}$ screening is to detect toxicity at an earlier stage such that significant visual loss may be prevented. As a result, when the American Academy of Ophthalmology (AAO) published revised guidelines on screening for HC retinopathy, fundus photography and fluorescein angiography were not recommended for screening. ${ }^{1}$

Screening tests felt to be appropriate to detect $\mathrm{HC}$ toxicity prior to onset of significant visual loss include 10-2 automated threshold visual fields, spectral domain optical coherence tomography (SD OCT), fundus autofluorescence (FAF), and multifocal electroretinogram (ERG).

We present two cases of bull's eye maculopathy associated with earlier $\mathrm{HC}$ toxicity manifest on near-infrared reflectance imaging and not detectable by ophthalmoscopy or fundus photography. A third case of more advanced $\mathrm{HC}$ toxicity showed an absence of bull's eye changes on near-infrared reflectance. We propose the hypothesis that bull's eye maculopathy on near-infrared imaging using a confocal scanning laser ophthalmoscope (cSLO) could be an indication of early HC toxicity and that this bull's eye change may disappear with more advanced HC toxicity. 


\section{Case report I}

A 68-year-old female without ocular complaints had been taking $\mathrm{HC}$ for 8 years for management of Sjögren's syndrome. She was $155 \mathrm{~cm}$ tall and weighed $68 \mathrm{~kg}$. Her daily dose based on ideal body weight was $7.34 \mathrm{mg} / \mathrm{kg} /$ day and her cumulative dose was $1,168 \mathrm{~g}$. She had no concomitant liver or renal disease.

Her visual acuities with correction were OD (right eye) $20 / 25+1$ and OS (left eye) 20/30+3.

$\mathrm{HC}$ toxicity was diagnosed based on taking a potentially toxic dose of $\mathrm{HC}$ in conjunction with a paracentral depression on visual field testing and SD OCT showing paracentral thinning of the outer retinal layers in both eyes (Figure 1A). Although ophthalmoscopy revealed no apparent pigmentary abnormalities, the near infrared reflectance images showed a bull's eye pattern of increased paracentral reflectance (Figure 1A). FAF revealed no evidence of hyperautofluorescence in either macula (Figure 1B). Color fundus photography (Figure 1C) and fluorescein angiography also failed to show a bull's eye maculopathy.

\section{Case report 2}

A 68-year-old female with systemic lupus erythematosus had been taking HC for 8 years. She was $160 \mathrm{~cm}$ tall and weighed $100 \mathrm{~kg}$. Her daily dose based on ideal body weight was $6.52 \mathrm{mg} / \mathrm{kg} /$ day with a cumulative dose of $1,168 \mathrm{~g}$. There was no concomitant renal or liver disease.

$\mathrm{HC}$ toxicity was diagnosed based on taking a potentially toxic dose of $\mathrm{HC}$ in conjunction with automated 10-2 visual fields showing central sensitivity loss (Figure 2A), multifocal ERG showing weak central waveform signals (Figure 2B), and SD OCT revealing thinning of the paracentral ellipsoid zone layer. Although ophthalmoscopy revealed no apparent bull's eye maculopathy, the near infrared reflectance image showed a bull's eye pattern of increased reflectivity (Figure 2C).
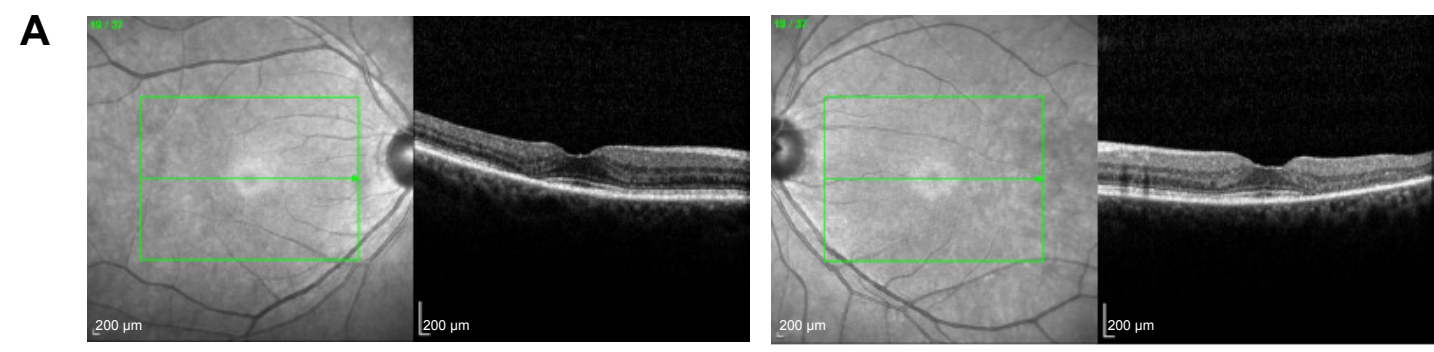

B
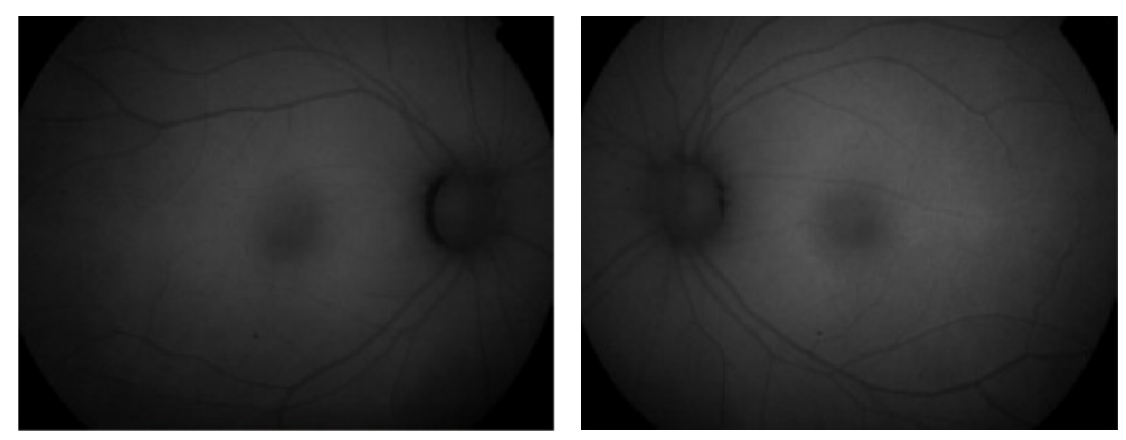

C
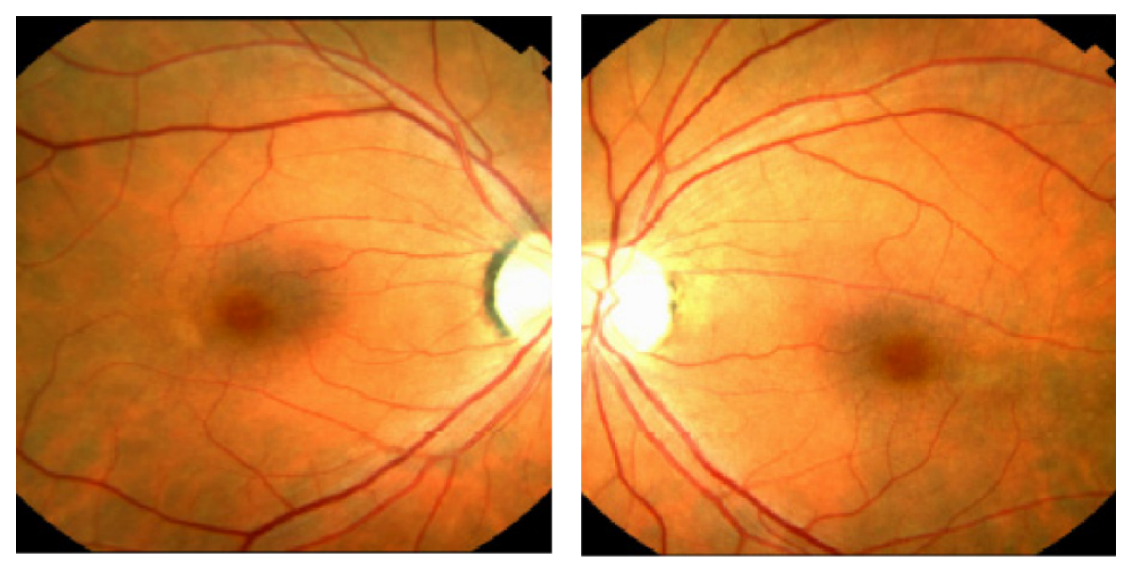

Figure I Case I.

Notes: Bull's eye maculopathy visible on near-infrared reflectance image (A) but not on autofluorescence (B) or fundus photography (C). 
A

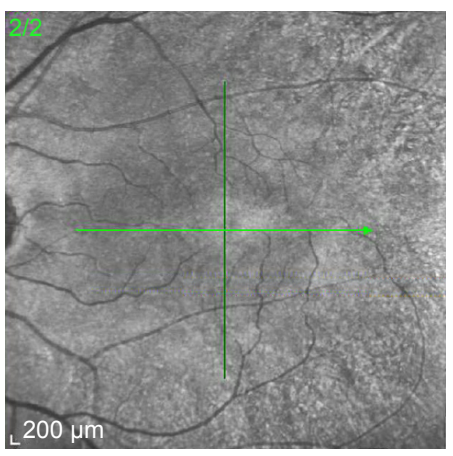

B

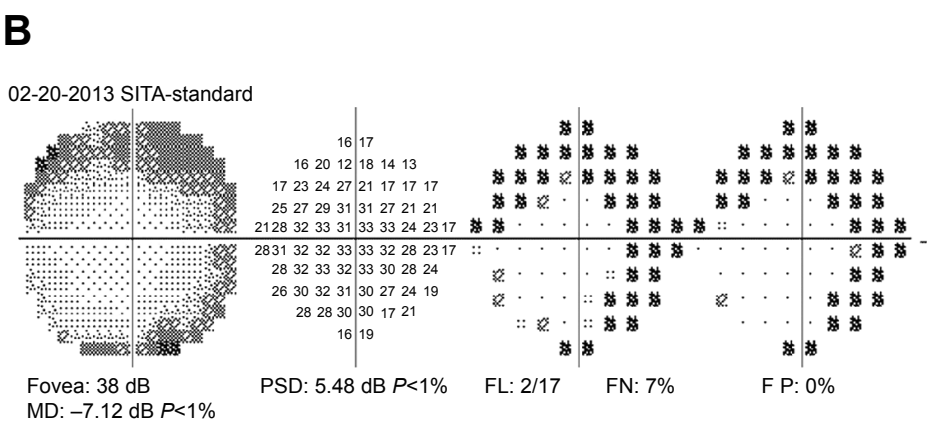

C

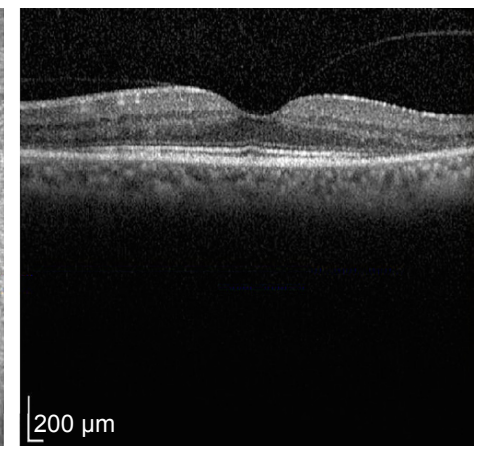

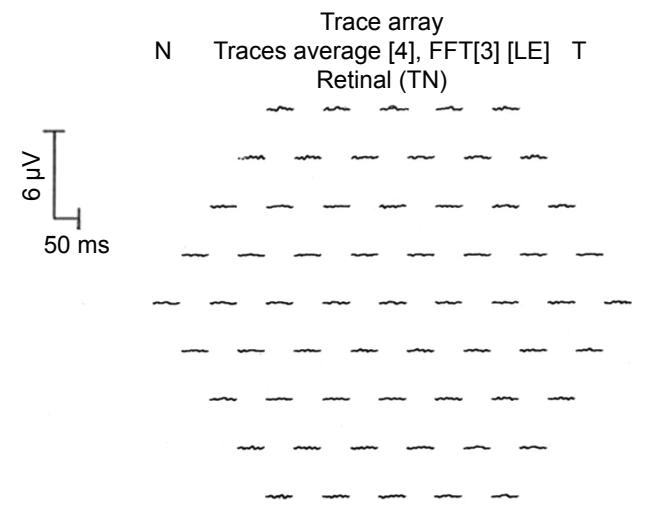

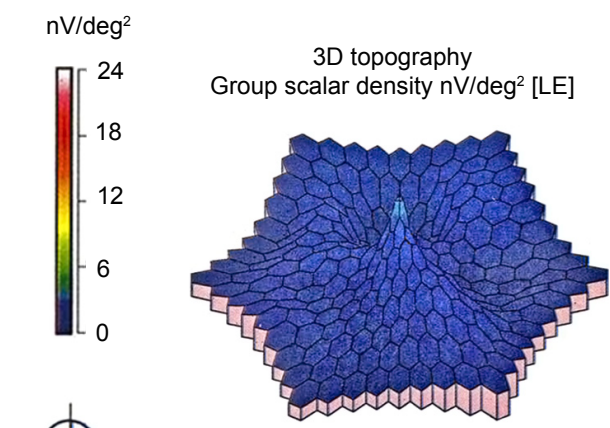

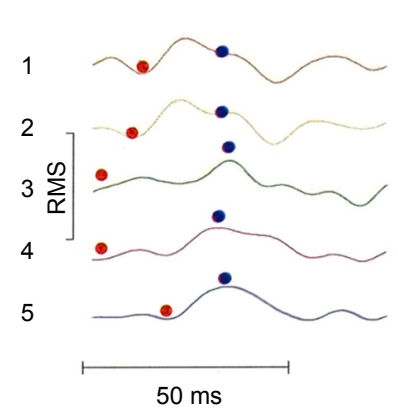

Group averages

Concentric rings [LE]

\begin{tabular}{|c|c|c|c|c|}
\hline \multicolumn{5}{|c|}{ Response normalized } \\
\hline Index & N1 ms & P1 ms & P1 nV/deg & R1/Rn P1 nV/d \\
\hline 1 & 20.0 & 39.1 & $4.6^{*}$ & 1.00 \\
\hline 2 & 17.5 & 39.1 & $2.3^{*}$ & 2.00 \\
\hline 3 & 10.0 & 40.8 & $1.1^{*}$ & 4.33 \\
\hline 4 & 10.0 & 38.3 & $1.6^{*}$ & 2.94 \\
\hline 5 & 25.8 & 39.9 & $1.7^{*}$ & 2.64 \\
\hline
\end{tabular}

Figure 2 Case 2

Note: Bull's eye maculopathy visible on near-infrared reflectance image. (A) Central visual field sensitivity loss and (B) multifocal electroretinogram showing weak central waveform signals with $(\mathbf{C})$ bull's eye maculopathy visible on near-infrared image.

\section{Case report 3}

A 38-year-old female with systemic lupus erythematosus had been taking $\mathrm{HC}$ for 11 years. She was $168 \mathrm{~cm}$ tall and weighed $60 \mathrm{~kg}$, so her actual body weight was less than her ideal body weight. Her daily dose based on actual body weight was $6.7 \mathrm{mg} / \mathrm{kg} / \mathrm{d}$ and she had a cumulative dose of $1,606 \mathrm{~g}$. There was no history of concomitant renal or liver disease.

$\mathrm{HC}$ toxicity was diagnosed based on taking a potentially toxic dose of $\mathrm{HC}$ in conjunction with diffuse central sensitivity loss on visual field testing (Figure 3B), a weak central waveform signal on multifocal ERG testing (Figure 3C), and a generalized loss of the ellipsoid zone through the fovea and parafoveal macula. The near infrared reflectance image did not show a bull's eye maculopathy (Figure 3A).

\section{Discussion}

Because automated threshold visual field testing with a white 10-2 pattern can detect parafoveal loss of visual sensitivity before pigmentary changes are seen on fundus examination, the AAO recommended such testing for screening for $\mathrm{HC}$ toxicity. Because abnormalities on multifocal ERG, SD OCT, and FAF tests can be more specific for HC toxicity, the AAO also recommended that one of these objective tests be performed in conjunction with visual field testing. 
A

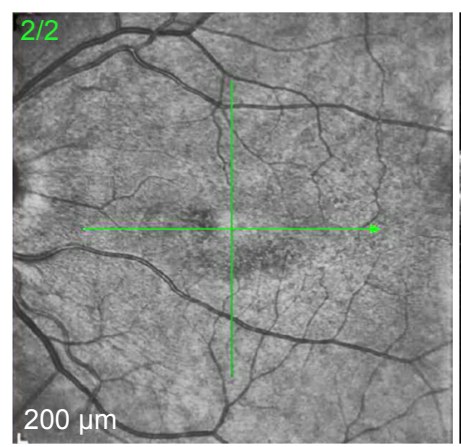

C

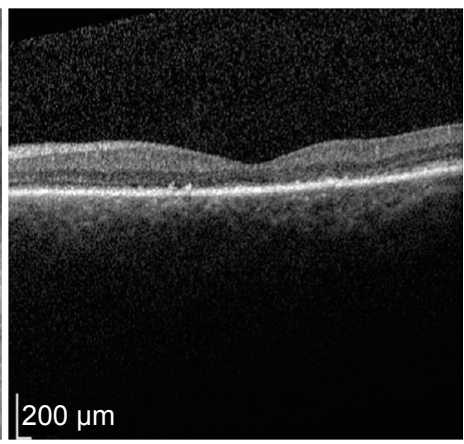

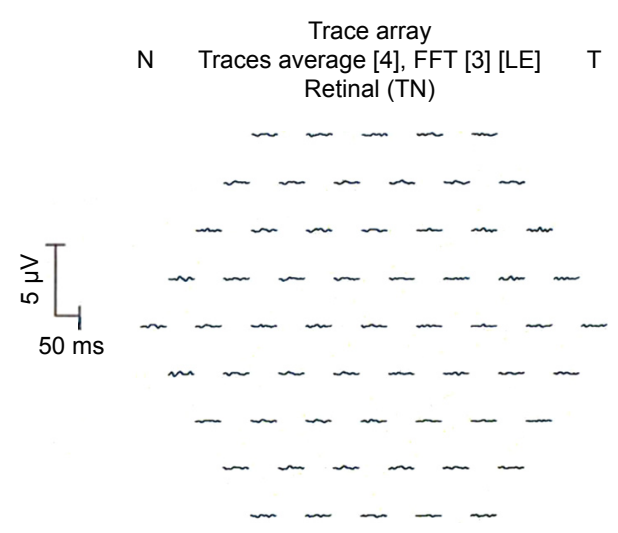

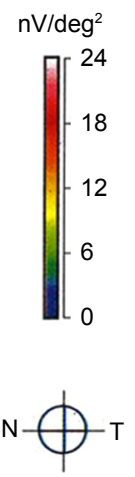

Group averages Concentric rings [LE]

\section{B}
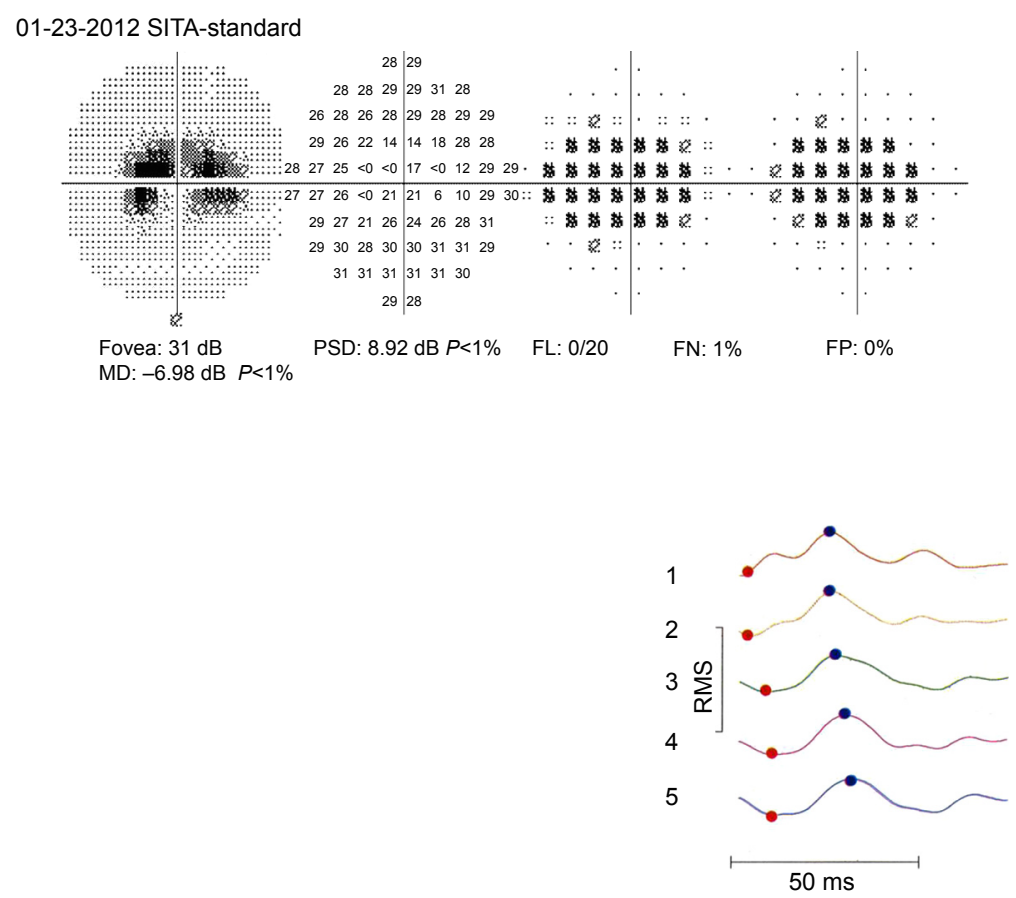

\begin{tabular}{|c|c|c|c|c|}
\hline \multicolumn{5}{|c|}{ Response normalized } \\
\hline Index & $\mathrm{N} 1 \mathrm{~ms}$ & $\mathrm{P} 1 \mathrm{~ms}$ & $\mathrm{P} 1 \mathrm{nV} / \mathrm{deg}^{2}$ & $\mathrm{R} 1 / \mathrm{Rn} \mathrm{P} 1 \mathrm{nV} / \mathrm{d}^{2}$ \\
\hline 1 & 10.0 & 31.6 & $14.1^{*}$ & 1.00 \\
\hline 2 & 10.0 & 31.6 & $6.5^{*}$ & 2.18 \\
\hline 3 & 15.0 & 33.3 & $3.7^{*}$ & 3.77 \\
\hline 4 & 16.6 & 35.8 & $3.5^{*}$ & 4.06 \\
\hline 5 & 16.6 & 37.4 & $1.7^{*}$ & 8.33 \\
\hline
\end{tabular}

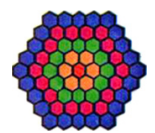

Figure 3 Case 3.

Notes: Advanced hydroxychloroquine toxicity with loss of outer retinal layers on spectral domain optical coherence tomography (A); marked loss of visual field (B); weak central waveform on multifocal electroretinogram (C); but without bull's eye maculopathy on near-infrared image.

SD OCT can detect early structural changes. Multifocal ERG can detect early paracentral functional abnormalities. ${ }^{2,3}$ Increased lipofuscin as a measure of RPE dysfunction is imaged by FAF. In the course of developing HC retinopathy, it is unclear when the structural changes, functional changes, or metabolic changes measured by these tests ensue, and individual cases of $\mathrm{HC}$ toxicity may show disparity in the appearance of abnormalities on these tests. ${ }^{4}$ However, the development of bull's eye maculopathy detected by ophthalmoscopy, fundus photography, or fluorescein angiography is typically a late finding already associated with significant vision loss. We report two cases of bull's eye maculopathy associated with asymptomatic $\mathrm{HC}$ toxicity in which the bull's eye maculopathy was detectable only with near-infrared reflectance. A case of more advanced, symptomatic HC toxicity, in which the bull's eye maculopathy was not apparent, suggests that bull's eye maculopathy visible on near-infrared reflectance imaging may be an additional objective sign of early $\mathrm{HC}$ toxicity and, of possible importance, that this sign may disappear with late $\mathrm{HC}$ toxicity.

This hypothesis has biologic plausibility. Although the mechanism by which $\mathrm{HC}$ causes toxicity is not well 
understood, the binding of the drug to melanin in the RPE may play a role. In contrast to flash photography using the visible spectrum of light, reflectance imaging using longer wavelength (near-infrared) light penetrates into the deeper layers of the retina to provide reflectance information at the level of the RPE. By selectively imaging the RPE, the nearinfrared reflectance images on the SD OCT may represent a logical objective technology to identify HC retinopathy. The boundaries of the bull's eye changes do not appear to colocalize with the margins of the ellipsoid zone disruption; so rather than being just an alternative indication of structural changes to the ellipsoid zone, it may be that the near-infrared reflectance may represent a biochemical change. ${ }^{5}$

In these cases, all of these fundus images demonstrating bull's eye maculopathy were obtained using a Spectralis SD OCT. The Spectralis SD OCT uses a cSLO with an $815 \mathrm{~nm}$ wavelength for image acquisition. The confocal technology has the advantage of not recording scattered light, and by doing so further improves the definition of information from the deeper layers of the retina. ${ }^{6}$ Since all of these images of bull's eye maculopathy reported here took place using a Spectralis SD OCT, the observations may not translate to the fundus images of other commercially available SD OCT machines.

In summary, near-infrared reflectance images with the cSLO provide information from the deeper layers of the retina at the level of the RPE where $\mathrm{HC}$ is absorbed. We provide observations that a bull's eye pattern on the near infrared reflectance image may be associated with early $\mathrm{HC}$ toxicity and hypothesize that the near infrared reflectance may provide an alternative objective measurement to screen for toxicity.

The sensitivity and specificity of all of the screening tests for HC are not firmly established. As screening tests, FAF and multifocal ERG are not as widely available in comprehensive ophthalmology practices as SD OCT and automated visual fields. Since the near-infrared reflectance image is obtained simultaneously with all Spectralis SD OCT images, it requires no additional technician time or significant physician effort to evaluate the near-infrared reflectance image. We hope that alerting clinicians to this possibility may provide an impetus for larger case series to either confirm or disprove this hypothesis. If the hypothesis is true, we believe that it may be easier for comprehensive ophthalmologists to recognize a pattern of bull's eye maculopathy more easily than the paracentral thinning of the ellipsoid zone seen on SD OCT, and as such, perhaps facilitate earlier detection of $\mathrm{HC}$ toxicity.

However, we emphasize that in the absence of further studies, we do not recommend using the near infrared image alone to provide any recommendations on altering the dose of HC. Pending larger studies, we believe it may be reasonable in such patients who show a bull's eye maculopathy on the near-infrared image to recommend an increased intensity of surveillance.

\section{Acknowledgment}

Keye L Wong has had full access to all of the data in the study and takes responsibility for the integrity of the data and the accuracy of the data analysis.

\section{Disclosure}

The authors report no conflicts of interest in this work.

\section{References}

1. Marmor MF, Kellner U, Lai TY, Lyons JS, Mieler WF; American Academy of Ophthalmology. Revised recommendations on screening for chloroquine and hydroxychloroquine retinopathy. Ophthalmology. 2011;118(2):415-422.

2. Nebbioso M, Livani ML, Steigerwalt RD, Panetta V, Rispoli E. Retina in rheumatic diseases: standard full field and multifocal electroretinography in hydroxychlroquine retinal dysfunction. Clin Exp Optom. 2011;94:276-283

3. Nebbioso M, Grenga R, Karavitis P. Early detection of macular changes with multifocal ERG in patients on antimalarial drug therapy. $J$ Ocul Pharmacol Ther. 2009;25:249-258.

4. Marmor MF, Melles RB. Disparity between visual feidls and optical coherence tomography in hydroxychloroquine retinopathy. Ophthalmology. 2014;121:1257-1262

5. Parodi MB, Iacono P, Del Turco C, Bandello F. Near-infrared fundus autofluorescence in subclinical Best vitelliform macular dystrophy. Am J Ophthalmol. 2014;158:1247-1252.

6. Elnser AE, Burns SA, Weiter JJ, Delori FC. Infrared imaging of subretinal structures in the human ocular fundus. Vision Res. 1996;36(1) 191-205.
Clinical Ophthalmology

\section{Publish your work in this journal}

Clinical Ophthalmology is an international, peer-reviewed journal covering all subspecialties within ophthalmology. Key topics include: Optometry; Visual science; Pharmacology and drug therapy in eye diseases; Basic Sciences; Primary and Secondary eye care; Patient Safety and Quality of Care Improvements. This journal is indexed on

\section{Dovepress}

PubMed Central and CAS, and is the official journal of The Society of Clinical Ophthalmology (SCO). The manuscript management system is completely online and includes a very quick and fair peer-review system, which is all easy to use. Visit http://www.dovepress.com/ testimonials.php to read real quotes from published authors. 